\title{
Effect of Eucommia leaf extract on water consumption in rats is mediated by the parasympathetic nervous system
}

Hirotaka Oikawa $^{1}$, Shouhei Miyazaki ${ }^{1}$, Haruka Nishide ${ }^{1}$, Shouko Nakamichi ${ }^{1}$, Nami Kawaguchi ${ }^{1}$, Tetsuya Hirata ${ }^{2}$, Taro Ueda ${ }^{2}$, Sansei Nishibe $^{3}$ and Takahiko Fujikawa ${ }^{1,4,5 *}$

${ }^{1}$ Faculty of Pharmaceutical Sciences, Suzuka University of Medical Science, 3500-3 Minamitamagaki-cho, Suzuka-city, Mie, 513-8670, Japan ${ }^{2} \mathrm{R} \& D$ Center, Kobayashi Pharmaceutical Co., Ltd. 1-30-3 Toyokawa, Ibaraki, Osaka 567-0057, Japan

${ }^{3}$ School of Pharmaceutical Science, Health Sciences University of Hokkaido, 1757 Kanazawa, Tobetsu-cho, Ishikari-gun, Hokkaido, 061-0293, Japan

${ }^{4}$ Division of Clinical Pharmacy and Pharmaceutical Sciences, Graduate School of Pharmaceutical Sciences. Suzuka University of Medical Science, 3500-3 Minamitamagaki-cho, Suzuka-city, Mie, 513-8670, Japan

${ }^{5}$ Mie University Graduate School/Faculty of Medicine, 2-174 Edobashi Tsu-city, Mie, 514-8507, Japan

\begin{abstract}
Eucommia leaf extract (ELE) is known to have a preventive effect on obesity. The effect of ELE partially depends on the inhibition of locomotor activity during inactive periods and indirect inhibition of food and water consumption. Moreover, it has been reported that suppression of the parasympathetic nervous system is involved in appetite suppression and that lipid metabolism is improved by ELE ingestion. However, there is no evidence that food and water consumption during the inactive period occur as a result of regulation of the parasympathetic nervous system. Therefore, in this study, the involvement of the parasympathetic nervous system was examined using water intake during the inactive period of rats as an index. Rats were fed a high-fat diet (35\% fat; HFD) or an HFD + ELE 5\% diet and were observed for 3 days during which pilocarpine $(10 \mathrm{mg} / \mathrm{kg} /$ day, intraperitoneally; i.p.) or saline (i.p.; used as a control) was administered daily. When compared with the control group, no effect of pilocarpine was observed in the dark phase (active period) or light phases (inactive period). The slightly increased water consumption of the HFD + ELE 5\% group was not affected by the administration of pilocarpine in the active period. However, the markedly decreased amount of water consumption in the inactive period that was due to ELE ingestion was restored to the control level by the administration of pilocarpine. These results suggest that the ingestion of ELE by rats causes changes in water consumption that are mediated by the parasympathetic nervous system.
\end{abstract}

\section{Introduction}

Du-zhong tea is a common drink ingested for health purposes. The tea is made by roasting the leaves of the Eucommia ulmoides tree. In recent years, the study of Eucommia leaf extract (ELE) has been focused on its efficacy in maintaining human health. Reports on the biogenic effectiveness of ELE ingestion have identified the following benefits: 1) antihyperlipidemic effects that reduce visceral fat and suppress elevated levels of triglyceride and cholesterol in the blood [17]; 2) effects on bone, such as preventing the onset of postmenopausal osteoporosis and promoting calcium accumulation in osteoblasts [810]; 3) antihypertensive effects that slowly decrease blood pressure by maintaining the flexibility of the blood vessels and promoting arterial smooth muscle relaxation $[2,11]$; 4) rapid eye movement sleep and non-rapid eye movement sleep inducing effect [12]; and 5) preventative effects on ulcerative colitis by asperuloside, one of the major components of ELE [13]. Thus, ELE promotes systemic health. Among the effects of ELE, many anti-obesity effects have been studied from the viewpoint of preventing lifestyle diseases. Therefore, we proposed that ELE ingestion might prevent the obesity caused by nocturnal eating, as ELE ingestion reduces food intake and water consumption during the light phase (i.e., inactive period) in rats $[7,14,15]$. On the other hand, it has been reported that appetite suppression due to the inhibition of the parasympathetic nervous system, which controls the gastrointestinal tract, is related to the suppression of feeding and the improvement of lipid metabolism by ELE ingestion [16,17]. However, it remains unknown whether decreased food intake and water consumption in the inactive period is mediated by the parasympathetic nervous system. Therefore, in this study, we examined the effect of ELE on the parasympathetic nervous system using water consumption during the inactive period of rats as an index.

Correspondence to: T Fujikawa, Laboratory of Molecular Prophylaxis and Pharmacology, Faculty of Pharmaceutical Sciences, Suzuka University of Medical Science, Minamitamagaki-cho, Susuka, Mie 513-8670, Japan. e-mail: fujikawa@suzuka-u.ac.jp

Key words: Eucommia leaf extract, parasympathetic nervous system, water intake, musculoskeletal disorders, Asperuloside

Special Issue: Application to prophylactic pharmacology of food

Hirotaka Oikawa

Assistant professor

Department of Pharmaceutical Sciences

Suzuka University of Medical Science

Japan

Takahiko Fujikawa

Professor

Suzuka University of Medical Science

Japan

Received: April 27, 2018; Accepted: April 29, 2018; Published: April 30, 2018 


\section{Materials and Methods}

\section{Animal rearing and water analysis}

Thirteen-week-old Sprague-Dawley (SD) male rats were used in the experiments. The rats were purchased from Japan SLC, Inc. The rats were reared for one week to condition them to the metabolic cage. After conditioning, the rats were observed for 3 days, during which time water consumption was assessed by a scale on the water bottle in the metabolic cage every day during the active period (19:00-07:00) and the inactive period (13:00-19:00 and 07:00-11:00). We used an MK-5000RQ metabolic measurement system (Muromachi Kikai Co., Ltd.) to measure water consumption. The light-dark cycle of the rat rearing room was in the light phase from 7 am to $7 \mathrm{pm}$ and in the dark phase for the remainder of each day. The experiments were conducted in accordance with the animal experiment guidelines established by the Suzuka Medical University Animal Experimental Ethics Committee, which approved the experimental plan $[1,15]$.

\section{Feeding regimes}

We used a high-fat diet (35\% fat; HFD) as the standard feed in this experiment. The HFD was purchased from Oriental Yeast Co., Ltd. as "MF powder" and contained 35\% lard [15]. A HFD diet containing 5\% ELE was fed to the ELE treatment group (HFD + ELE 5\%). The ELE used in the study was purified and provided by Kobayashi Pharmaceutical Co., Ltd. In contrast, only an HFD was fed to the control group (HFD cont.) [15]. The treatments for the two groups continued from rearing to the end of the experiment.

\section{Drug administration}

After rearing the HFD and HFD + ELE 5\% groups, pilocarpine treatment rats were randomly selected from both groups. The selected rats were then intraperitoneally (i.p.) administered $10 \mathrm{mg} / \mathrm{kg} / \mathrm{day}$ pilocarpine at 19:00 daily for 3 days. Likewise, saline treatment rats were selected as a control for the pilocarpine group. Control group rats were administered saline on the same time schedule as pilocarpine.

\section{Results and discussion}

According to previous reports, ELE has an inhibitory effect on the parasympathetic nervous system [16,17]. Therefore, we used pilocarpine as a control reagent for ELE in this study to demonstrate anticholinergic effects as a phenotype. Pilocarpine was administered in a dose that did not affect the water consumption of the control HFD group for the purpose of examining the cholinergic effect at the biological level (Figure 1). After observing the water intake of rats fed with HFD or HFD + ELE 5\% for 3 days, it was found that water consumption tended to increase in the dark phase (i.e., the active period) of the rats that had ingested ELE, though the difference was not statistically significant (Figure 1a). On the other hand, water consumption in the light phases (i.e., the inactive period) was markedly decreased following ELE ingestion (Figure 1b). In the rats fed an HFD, this change correlated with the change in the rats' water consumption [15]. The results of a previous report have shown that ELE significantly altered water consumption after the 6th day of administration in the active period and after the 4th day in the inactive period [15]. The present study assessed average water consumption for only 3 days. This was because we determined that extended pilocarpine administration would be burdensome to the rats. When the parasympathetic nervous system is elevated more than necessary, systemic perspiration is promoted. Therefore, long-term administration of pilocarpine may cause side effects such as abnormal perspiration. Thus, we limited the administration of pilocarpine to 3 days to prevent such side effects. In general, it is known that the sympathetic and parasympathetic nervous systems are balanced during the active period. As dry mouth is a known side effect of anticholinergic drugs [18], it is thought that water consumption increased due to the anticholinergic action of ELE (Figure 1a). On the other hand, water consumption during the inactive period is thought to have decreased due to ELE ingestion. It is known that the parasympathetic nervous system dominates during rest [19]. It speculated that the parasympathetic nervous system is predominant at rest because water consumption is 10 times lower during the inactive period than during the active period (Figure 1). As parasympathetic nerve activity decreases, gland secretion also decreases [20]. However, it should be considered that the effect of ELE on water consumption was different between the active and inactive periods (Figure 1). It has already been reported that ELE has an appetite suppressing effect by inhibiting the parasympathetic nervous system, which controls the gastrointestinal tract [16]. On the other hand, chlorogenic acid, one of the major components of ELE, is known to potentiate parasympathetic nerves [21]. From these reports and our results, it can be assumed that the effect of ELE on the parasympathetic nervous system may be reversed between the active and inactive periods due to diurnal variation. This assumption explains our findings showing that water consumption in the inactive period was relatively decreased in the ELE ingestion group (Figure 1b). Rats that had ingested ELE and had pilocarpine administered during the active period showed a tendency of increased water consumption compared with the rats that had ingested only pilocarpine, though the difference was not statistically significant (Figure 1a). On the other hand, in this study, the decreased water consumption in the inactive period of rats that had ingested ELE and the improvement of this effect following pilocarpine was significant (Figure 1b). During the inactive period, during which parasympathetic activity is dominant, ELE affected the parasympathetic nerves; pilocarpine, as a parasympathomimetic drug, neutralized the action of ELE during this period. During the inactive period, in which the parasympathetic nervous system was predominant, pilocarpine was administered in addition to parasympathomimetic ELE; as a result, the drinking behavior associated with the sympathetic activation might have been caused reflectively to maintain vital homeostasis. However, these considerations do not come out as predictions. Therefore, future studies should assess the autonomic nervous system using electrocardiography, and indices such as urine volume change and osmotic pressure change should be incorporated to render more reliable results. In addition, since it has been predicted that the main components of ELE have different actions, a more detailed examination of each component is required. It would be very interesting to investigate the relative results found in the active period and the inactive period in terms of the action of various ELE components, and it is important to consider the diurnal variation of living organisms and the timing of food ingestion. In this study, ELE administration reduced water consumption during the inactive period. In addition, it is known that nocturnal eating and drinking, especially alcohol ingestion, occur frequently in obese people [22]. Therefore, our results suggest that ELE ingestion may prevent nocturnal eating and drinking and may therefore contribute to a reduction in obesity.

\section{Acknowledgments}

The authors are grateful to M. Nakagawa for technical assistance and statistical analysis and S. Nakao for animal care. This study was supported by a research grant from the Japanese Society of Eucommia. The Eucommia leaf extract samples used in this study were provided by KOBAYASHI Pharmaceutical Co., Ltd. (Osaka, Japan). 


\section{a \\ Dark phase (active period)}

(19:00-7:00)

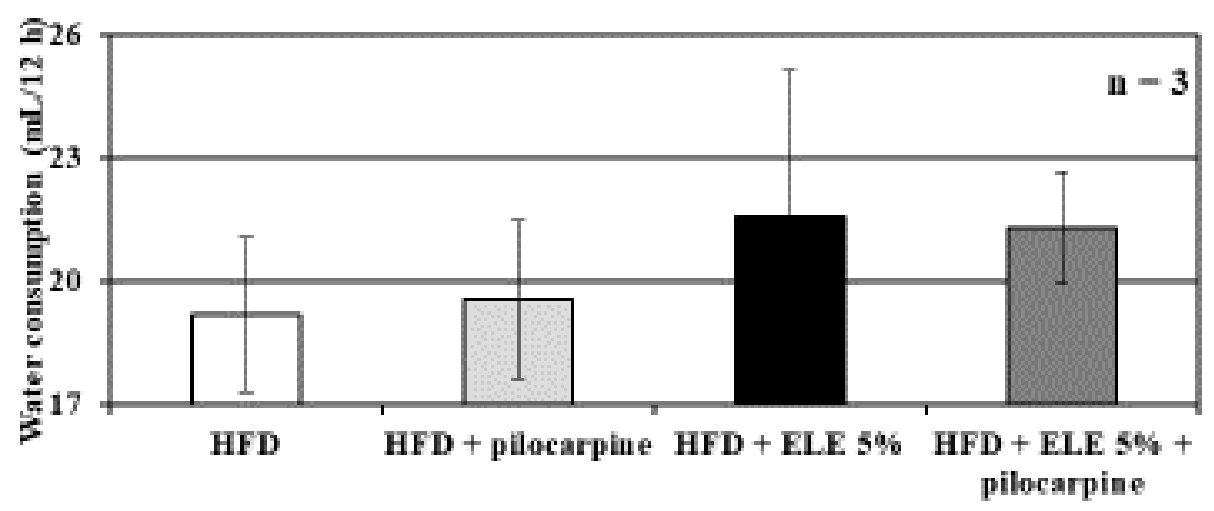

b Light phases (inactive period)

$(13: 00-19: 00+7: 00-11: 00)$

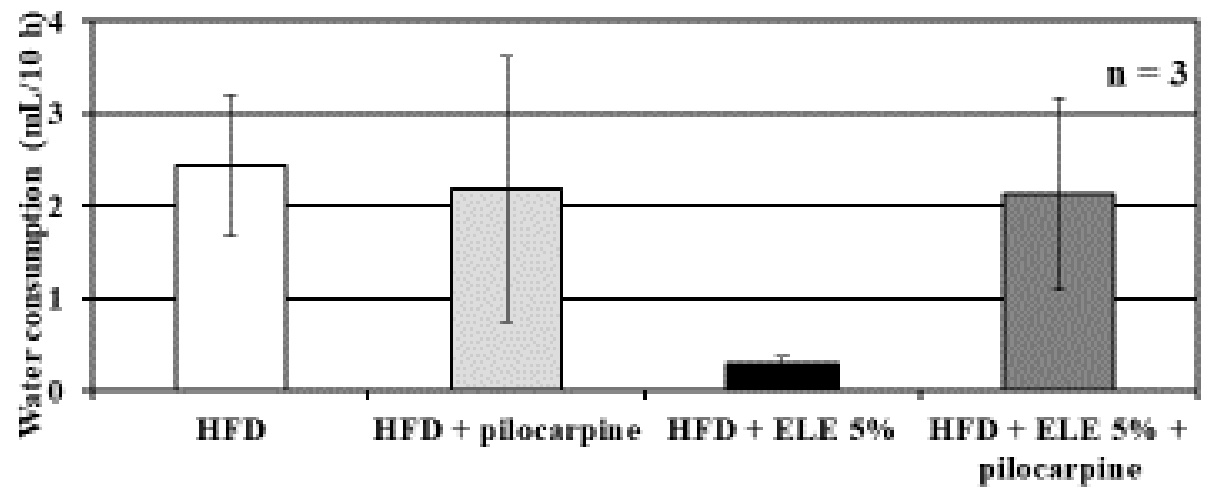

Figure 1. Effect of ELE ingestion on the parasympathetic nervous system. This experiment used water consumption to measure the effect of ELE on the parasympathetic nervous system. (a) Rats were divided into 4 groups, as follows: HFD, HFD + pilocarpine, HFD + ELE 5\%, and HFD + ELE 5\% + pilocarpine. Water consumption in the dark phase (i.e., the active period of the rat) between 19:00 and 7:00 was measured using a water bottle scale in a metabolic cage. Water consumption in the HFD ingestion group and the HFD + ELE 5\% ingestion group were not affected by pilocarpine administration. (b) Rats were divided into 4 groups, as in (a). Water consumption in the light phase (i.e., the inactive period of the rat) between 13:00 and 19:00 and between 7:00 and 11:00 was measured using a water bottle scale in a metabolic cage. The water consumption of the HFD ingestion group was not affected by pilocarpine administration. However, in the HFD + ELE 5\% ingestion group, water consumption was markedly increased by pilocarpine administration. All results were expressed as the mean \pm S.E., and statistical significance was determined by one-way analysis of variance (ANOVA) with a Tukey and/or Dunnett's test.

\section{Conflicts of interest}

There are no conflicts of interest to declare.

\section{References}

1. Fujikawa T, Hirata T, Wada A, Kawamura N, Yamaguchi Y, et al. (2010) Chronic administration of Eucommia leaf stimulates metabolic function of rats across several organs. Br J Nutr 104: 1868-1877. [Crossref]

2. Fujikawa T, Hirata T, Hosoo S, Nakajima K, Wada A, et al. (2012) Asperuloside stimulates metabolic function in rats across several organs under high-fat diet conditions, acting like the major ingredient of Eucommia leaves with anti-obesity activity. J Nutr Sci 1: e10. [Crossref]

3. Hussain T, Tan B, Liu G, Oladele OA, Rahu N, et al. (2016) Health-Promoting Properties of Eucommia ulmoides: A Review. Evid Based Complement Alternat Med 2016: 5202908. [Crossref]

4. Choi MS, Jung UJ, Kim HJ, Do GM, Jeon SM, et al. (2008) Du-zhong (Eucommia ulmoides Oliver) leaf extract mediates hypolipidemic action in hamsters fed a high-fat diet. Am J Chin Med 36: 81-93. [Crossref]

5. Metori K, Ohashi S, Takahashi S, Tamura T (1994) Effects of Du-Zhong leaf extract on serum and hepatic lipids in rats fed a high-fat diet. Biol Pharm Bull 17: 917 920. [Crossref]
6. Jin K, Amitani Y, Zamami Y, Takatori S, Hobara N, et al. (2010) Ameliorative effect of Eucommia ulmoides Oliv. leaves extract (ELE) on insulin resistance and abnormal perivascular innervation in fructosedrinking rats. J Ethnopharmacol 128: 672-678. [Crossref]

7. Kobayashi Y, Hiroi T, Araki M, Hirokawa T, Miyazawa M, et al. (2012) Facilitative effects of Eucommia ulmoides on fatty acid oxidation in hypertriglyceridaemic rats. $J$ Sci Food Agric 92: 358-365. [Crossref]

8. Zhang W, Fujikawa T, Mizuno K, Ishida T, Ooi K, et al. (2012) Eucommia leaf extract (ELE) prevents OVX-induced osteoporosis and obesity in rats. Am J Chin Med 40: 735-752. [Crossref]

9. Zhang Z, Liu ZG, Li C, Hu SJ, Liu L, et al. (2009) Du-Zhong (Eucommia ulmoides Oliv.) cortex extract prevent OVX-induced osteoporosis in rats. Bone 45: 553-559. [Crossref]

10. Oikawa H, Miyazaki S, Nishida K, Zhang W, Hirata T, et al. (2017) Promotion of osteoblastic Ca2+ accumulation by Eucommia leaf extract. Glob Drugs Therap 3: 2.

11. Hosoo S, Koyama M, Watanabe A, Ishida R, Hirata T, et al. (2017) Preventive effect of Eucommia leaf extract on aortic media hypertrophy in Wistar-Kyoto rats fed a high-fat diet. Hypertens Res 40 (6): 546-551. [Crossref]

12. Miyazaki S, Oikawa H, Nakamichi S, Hirata T, Yamasaki H, et al. (2017) Aroma of Eucommia leaf extract (ELE) causes reduced locomotor activity and increased NREM sleep, acting like the partially related factors of oral ELE's effects with locomotoractivity-dependent-increase in NREM- and REM-sleep. Glob Drugs Therap 3: 2. 
13. Hiramoto K, Goto K, Sekijima H, Ooi K, Hirata T, et al. (2017) The preventive effects of asperuloside administration on dextran sodium sulfate-induced ulcerative colitis in mice. Glob Drugs Therap 3: 2.

14. Oikawa H, Nakamichi S, Nishide H, Kawaguchi M, Zhang W, et al. (2016) Engagement of the aroma in feeding suppression behavior of Eucommia leaf. Aroma Res 17: 74-79.

15. Oikawa H, Miyazaki S, Zhang W, Nishide H, Nakamichi S, et al. (2017) The characteristic taste of Eucommia leaf extract as the additive of a weight-loss and the constipation improvement is not associated with suppression of the feeding behavior of the fast rats with the extract. Glob Drugs Therap 3: 2 .

16. Horii Y, Tanida M, Shen J, Hirata T, Kawamura N, et al. (2010) Effects of Eucommia leaf extracts on autonomic nerves, body temperature, lipolysis, food intake, and body weight. Neurosci Lett 479: 181-186. [Crossref]

17. He X, Wang J, Li M, Hao D, Yang Y, et al. (2014) Eucommia ulmoides Oliv.: ethnopharmacology, phytochemistry and pharmacology of an important traditional Chinese medicine. J Ethnopharmacol 151: 78-92. [Crossref]
18. Tiisanoja A, Syrjälä AM, Komulainen K, Lampela P, Hartikainen S, Taipale H, et al. (2018) Anticholinergic burden and dry mouth among Finnish, community-dwelling older adults. Gerodontology 35: 3-10. [Crossref]

19. da Silva SA, Guida HL, Dos Santos Antonio AM, de Abreu LC, Monteiro CB, et al. (2014) Acute auditory stimulation with different styles of music influences cardiac autonomic regulation in men. Int Cardiovasc Res $J$ 8: 105-110. [Crossref]

20. Proctor GB, Carpenter GH. (2014) Salivary secretion: mechanism and neura regulation. Monogr Oral Sci 24: 14-29. [Crossref]

21. Park I, Ochiai R, Ogata H, Kayaba M, Hari S, et al. (2017) Effects of subacute ingestion of chlorogenic acids on sleep architecture and energy metabolism through activity of the autonomic nervous system: a randomised, placebo-controlled, double-blinded cross-over trial. Br J Nutr 17: 979-984. [Crossref]

22. Tikkinen KA, Auvinen A, Huhtala H, Tammela TL (2006) Nocturia and obesity: a population-based study in Finland. Am J Epidemiol 163: 1003-1011. [Crossref]

Copyright: $\odot 2018$ Oikawa H. This is an open-access article distributed under the terms of the Creative Commons Attribution License, which permits unrestricted use, distribution, and reproduction in any medium, provided the original author and source are credited. 